\title{
Cost-utility Analysis of Extensile Lateral Approach Versus Sinus Tarsi Approach in Sanders type II/III Calcaneus Fractures
}

\section{Zihua Li}

Shanghai Tongji Hospital; Tongji University School of Medicine

\section{Xinbo Wu}

Shanghai Tongji Hospital; Tongji University School of Medicine

\section{Haichao Zhou}

Shanghai Tongji Hospital; Tongji University School of Medicine

\section{Shaochen Xu}

Shanghai Tongji Hospital; Tongji University School of Medicine

\section{Fajiao Xiao}

Shanghai Tongji Hospital; Tongji University School of Medicine

Hui Huang

Shanghai Tongji Hospital; Tongji University School of Medicine

Yunfeng Yang ( $\nabla$ dr_yangyf123@163.com )

\section{Research article}

Keywords: cost-utility analysis, calcaneus fracture, extensile lateral approach, sinus tarsi approach

Posted Date: July 31st, 2020

DOI: https://doi.org/10.21203/rs.3.rs-17554/v3

License: (a) This work is licensed under a Creative Commons Attribution 4.0 International License. Read Full License

Version of Record: A version of this preprint was published on September 18th, 2020. See the published version at https://doi.org/10.1186/s13018-020-01963-5. 


\section{Abstract}

Background. Extensile lateral approach had been recognized as the gold standard technique for displaced intra-articular calcaneus fractures (DIACFs) while sinus tarsi approach had been increasingly valued by surgeons and comparative clinical outcome was shown in both techniques. Appropriate decisions could be made by the clinicians with the help of cost-utility analysis (CUA) about optimal healthcare for type II/III calcaneus fracture.

Method. A single-center, retrospective study was conducted in which basic characteristics, clinical outcomes and health care costs of 109 patients had been obtained and analyzed. Changes in healthrelated quality of life (HRQoL) scores, validated by EuroQol five-dimensional-three levels (EQ-5D-3L), were used to enumerate quality-adjusted life-years (QALYs). Cost-effectiveness was determined by the incremental cost per QALY.

Results. 109 patients were enrolled in our study including 62 in the ELA group and 47 in the STA group. There were no significant differences between these two groups in mean total cost, laboratory and radiographic evaluation expense, surgery, anesthesia and antibiotic expense. The expense of internal fixation materials $(\$ 3289.0 \pm 543.9)$ versus $(\$ 2630.6 \pm 763.7)$ and analgesia $(\$ 145.8 \pm 85.6)$ versus $(\$ 102.9 \pm 62.7)$ in ELA group were significantly higher than in the STA group $(P \otimes .001, P=.008$, respectively). Visual Analogue Scale (VAS) scores showed significant difference at postoperative 3 and 5 days ( $P \otimes .001$ ). American Orthopaedic Foot and Ankle Society (AOFAS) ankle-hindfoot scores and the Bohlers' and Gissane angle showed no significant differences between the two groups before and after the operation. The cost-effectiveness ratios of ELA and STA were $\$ 8766.8 \pm 2835.2 / Q A L Y$ and $\$ 7914.9 \pm$ 1822.0/QALY respectively and incremental cost-effectiveness ratio (ICERs) of ELA over STA was \$32110.00/QALY, but both showed no significant difference.

Conclusion. Both ELA and STA techniques are effective operative procedures for the patients with calcaneus fracture. Moreover, STA seems to be more reasonable for its merits including less postoperative pain, and less expense of analgesia as well as internal fixation materials.

\section{Background}

Calcaneus fracture is the most commonly happened one in tarsal bone, accounting for $2 \%$ of all fractures ${ }^{1-3}$ and $75 \%$ of which are displaced intra-articular calcaneus fractures (DIACFs). ${ }^{4,5}$ According to the classification system introduced by Sanders in 1993, calcaneus fracture is classified into four types based on the number and location of articular fragments seen on coronal and axial Computed Tomography (CT) ${ }^{6,7}$ Nonoperative and operative treatments are the two traditional managements of calcaneus fractures. However, patients with conservative treatment may develop posttraumatic arthritis, chronic heel deformity and malalignment of mechanical axis of the limb, which may seriously affect the quality of life. ${ }^{8,9}$ Surgical treatment is therefore necessary, and open reduction and internal fixation (ORIF) via extensile lateral approach (ELA) have been considered as the gold standard in the 
management of type II/III calcaneus fracture for its clear visualized for reduction and favorable outcomes in long-term. ${ }^{10-13}$ However, some articles report multiple complications of ELA including hematoma, wound slough, dehiscence and infection and the occurrence rate is approaching $33 \% .{ }^{14-18}$ Many minimally invasive approaches including closed reduction, percutaneous screws fixation and modified lateral approach (minimal invasive sinus tarsi approach) have been introduced in order to reduce the incidence of surgical complications, ${ }^{19-23}$. Many investigators and surgeons have advocated using sinus tarsi approach (STA) for its lower reported wound complications rate, lower visual analog pain scale levels, comparative clinical outcomes as well as increased overall satisfaction, ${ }^{24-26}$ and there has been a trend toward this technique in recent times. However, the optimal surgical approach for accurate reduction of DIACFs still remains controversial.

Owing to persistent pain, stiffness and gait abnormalities, DIACFs may result in permanent disability and reduction of quality of life. ${ }^{5,27-29}$ Additionally, DIACFs are more frequently happened in the most economically active population such as adults and adolescents, which undoubtedly lay high socioeconomic costs for its difficulties to return to work. ${ }^{30,31}$ Therefore, with growing pressure to reduce costs while high-quality care is still delivered, cost-effective research in calcaneus fracture is becoming increasingly important to discuss, which may help clinicians make appropriate surgical strategies and reach optimal health care. ${ }^{32}$

Cost-utility analysis (CUA), which belongs to effectiveness analysis, evaluates the outcome in units of utilities standing for quantity and quality of life. ${ }^{33,34}$ Alterations in health-related quality of life (HRQOL) scores validated by EuroQol five-dimensions three-levels (EQ-5D-3L) were applied to enumerate qualityadjusted life years (QALYS), which presents the result of CUA. In addition, incorporated with cost information, an incremental cost-effectiveness ratio (ICER) can be used to enumerate between two techniques and display a cost per QALY value: lower figure represents more cost-effective strategy. ${ }^{35,36}$

To the best of our knowledge, there had not been any cost-effectiveness research comparing the two approaches of calcaneus fractures conducted in China Therefore, the purpose of this study was to assess the cost and cost-effectiveness of two techniques for treatment of sanders type II/III in calcaneus fractures and thus it may help us make optimal clinical decisions.

\section{Method}

General Information. We conducted a retrospective review of 109 patients (62 in the ELA group and 47 in the STA group) who had been diagnosed as type II/III calcaneus fracture and had undergone ORIF surgery via ELA or STA from January 2013 to October 2018. Basic demographic and clinical characteristic of the patients were obtained including age, gender, type of fracture, anesthesia, the length of hospital stay and follow-up time.

Inclusion and Exclusion Criteria. Participants were enrolled if they met the following eligibility criteria: (1) DIACFs classified as sanders type II/III; (2) closed and fresh fractures; (3) fractures that underwent 
procedures via ELA or STA; (4) follow-up period greater than 1 year. The exclusion criteria were as follows: (1) fractures classified as sander I or IV; (2) malunion, nonunion, open, and bilateral fractures; (3) associated or multiple trauma; (4) severe systemic diseases and commodity.

Clinical Assessment. The pain was measured using Visual Analogue Scale (VAS) with the score ranged from 0 to 10 points indicating no pain to worst pain. The VAS scores were collected at pre-operation, postoperative 3 days, 5 days and 7 days. Functional outcome was measured by American Orthopaedic Foot and Ankle Society (AOFAS) ankle-hindfoot scores at pre-operation, postoperative 3 months and at final follow up with the scores ranging from 0-100 points of pain, function and alignment. Radiographic including Bohler's angle and Gissane angle were measured on fluoroscopic images at pre-operation, postoperative 3 months and at final follow-up time. The specific radiographic views including the anteroposterior (AP), lateral, axial projections and CT-scans were collected preoperatively. Based on the anatomic characteristic of calcaneus fractures shown in preoperative radiographs and $\mathrm{CT}$, surgeons could attain the information of the type, level, locations of the fractures and measure height, width, Bohler's and Gissane angle of the calcaneus.

Cost and Cost-utility Analysis. The medical and financial records of patients including the expense of total costs, laboratory and radiographic evaluation, surgery, anesthesia, analgesia, internal fixation materials and antibiotic were obtained from our hospital's medical and financial information center. However, indirect costs including miss time from work or decreased productivity, rehabilitation, further consultation, transportation were not enumerated because each patients' situation and the employment status varied. All costs were converted into U.S. dollars (\$) at their value during January 2020.

Clinical outcome was monitored by recording health-related quality of life (HRQOL) scores before and after operation with a score ranging from -0.11 to 1.0 and 1.0 indicates full health. Validation of HRQoL scores were converted by EQ-5D-3L including five dimensions: mobility, self-care, activities of daily life, pain and anxiety/depression and each depicted by three level from no, mild or moderate to severe problems. Thus, this depictive system includes 243 combinations, or health states. Combining HRQoL index and time were used to estimate QALYs, which are enumerated for the area under the curve by trapezoidal method.

The CUA was conducted from the healthcare perspective and was presented by the cost-effectiveness ratio (CER) and incremental cost-utility ratio (ICER). An annual discount of $3 \%$ was adjusted to QALYs and mean total costs.

\section{See formula 1 in the supplementary files.}

Different discount rates (0\% and 5\%) were used to mean total costs and QALYs for sensitivity analysis.

\section{Surgical Technique}

The decisions including the surgical procedures, type of the incision: ELA or STA and type of the implants were made by senior surgeons based on the anatomic characteristic of the fractures and their preference. 
There were three experienced surgeons included in total, and were coded as A, B and C in this study. They were all learned from the same professor and trained in the same place, and therefore the surgeons can perform the procedures with equivalent effectiveness and low complication rate.

\section{Extensile Lateral Approach}

Patients with a tourniquet at the thigh were under epidural, local, general or subarachnoid block anesthesia in the lateral position. The incision of ELA, which originated perpendicularly from $5 \mathrm{~cm}$ over lateral malleolus or the midpoint between the fibula and Achilles tendon and stopped at the base of $5^{\text {th }}$ metatarsal, was performed by experienced surgeons followed the way which was depicted by Benirshcke and Sangeorzan. With the flap held by several 2.0-mm Kirschner wires, this incision allowed the visualization of the lateral wall of calcaneus and subtalar joint and anatomic reduction was achieved directly under the guidance of C-arm fluoroscopy. Consequently, the length, height and width of the calcaneus were restored (Fig.1).

\section{Sinus Tarsi Approach}

Patients with a tourniquet at the thigh were under epidural, local, general or subarachnoid block anesthesia in the lateral position. The incision was performed along a line from the tip of the lateral malleolus to the base of the fourth metatarsal and its length was approximately $3-5-\mathrm{cm}$. This incision allowed the visualization of the posterior facet, anterior process and even the calcaneocuboid joint with the calcaneofibular ligament dissected and the extensor brevis muscle elevated. Two 2.5-mm Schanz screw were inserted percutaneously into the calcaneal tuberosity and talus from medial to lateral, and tibia distraction device (Johnson \& Johnson, USA) was applied to correct the deformity and restore the length, height, and width of the calcaneus with the guidance of C-arm fluoroscopy (Fig.2).

\section{Statistical Analysis}

The Kolmogorov-Smirnov test was applied to examine the normality of all variables. The between-group differences were compared by Student's $t$-test for normal distribution variables (mean \pm standard deviation) or a Mann-Whitney $U$ test for abnormal distribution variables (median (25\% quartile-75\% quartile)) or a chi-square test for categorical variables (n (\%)). The differences in the longitudinal changes of the Bohler's, Gissane angle and VAS scores between ELA and STA group were compared by applying non-parametric test. All analyses were performed using SPSS 20.0 software (SPSS, IL, USA). Differences were considered to be statistically significant when $P$ value was less than .05 .

\section{Results}

\section{Patient Information}

A total of 109 patients were enrolled in our research, including 62 in ELA group (45.40 \pm 12.91 years old, $82.26 \%$ male) and 47 in STA group (49.92 \pm 14.98 years old, $80.85 \%$ male). In the ELA group, 36 were 
classified as Sanders type II and 26were Sanders type III whereas in the STA group 28were classified as Sanders type II and 19 were Sanders type III. A sum of 52 in the ELA group underwent spinal anesthesia and 10 underwent general anesthesia whereas among the patients in the STA group, 40 underwent spinal anesthesia and 7 were under general anesthesia. The length of hospital stay and follow-up time were comparative between the ELA group and STA group. There were no significant differences in age, gender, fracture type, antibiotics, anesthesia, the length of hospital stay and the follow-up time between these groups (Table 1).

\section{Clinical outcome}

All the clinical outcomes including VAS scores, AOFAS scores, the Bohler's angle and the Gissane angle were presented in Table 2 . Results showed that VAS score was significantly different at postoperative 3 and 5 days between the two groups $(P \otimes .001, P=.002$, respectively). However, there were no significant difference in VAS scores before operation and at the final follow-up time between these two groups ( $P$ $=.151, P=.693$, respectively). AOFAS scores improved greatly 3 months after surgery, from $50.0 \pm 8.0$ to $80.3 \pm 6.3$ in the ELA group and from $51.2 \pm 7.9$ to $81.4 \pm 5.6$ in the STA group, but there showed no significant difference between two groups at these time points ( $P=.413, P=.325$, respectively). Similarly, the Bohler's angle and the Gissane angle were significantly improved 3 months after surgery, from 13.6 degrees \pm 7.3 to 30.2 degrees \pm 7.6 (P凶.001) and from 102.8 degrees \pm 14.2 to 130.0 degrees $\pm 9.2(P \otimes .001)$ in the ELA group whereas in the STA group, the Bohler's angle changed from 16.0 degrees \pm 7.4 to 28.2 degrees $\pm 7.1(P \bigotimes .001)$ and the Gissane angle changed from 102.1 degrees \pm 12.5 to 126.0 degrees \pm 7.5 ( $P$ ه.001). There showed no significant difference in the Bohler's and the Gissane angle before operation $(P=.086, P=.0530)$ and at postoperative 3 months $(P=.920, P=.255)$. Moreover, there were no significant differences among AOFAS scores, the Bohler's angle and the Gissane angle at final follow-up between these two groups $(P=.113, P=.065, P=.139$, respectively $)$.

\section{Health Care Costs}

Table 3 summarizes the itemized mean costs of ELA and STA groups. There were no significantly statistical differences between these two groups in the total costs and expense of laboratory and radiographic evaluation, surgery, antibiotics and anesthesia. However, the expense of internal fixation materials and analgesia exists significant difference between the two groups $(P \otimes .001, P=.008$, respectively).

\section{Cost-utility Analysis}

Changes in HRQoL scores validated by EQ-5D-3L in the two groups are presented as median (25\% quartile, $75 \%$ quartile) in Table 2. The average baseline HRQoL scores of the ELA and STA group were similar (median 0.63; range, 0.57-0.68) and (median 0.59; range, 0.52-0.68), respectively, $P=.246$ ). The average HRQoL scores at 1 year (0.92 in the ELA group and 0.93 in STA group) showed no significant difference $(P=.755)$. The mean gained QALYs of the ELA and STA groups are 0.77 (range, $0.73-0.80)$ and 
0.75 (range, 0.69-0.79), respectively. However, no significantly statistical difference exists in gained QALYS $(P=.250)$.

The consequences of CUA were described on Table 4. The CERs of ELA and STA groups were $\$$ 8766.8 $\pm 2835.2 /$ QALY and $\$ 7914.9 \pm 1822.0 /$ QALY $(P=.245)$, respectively. The difference between the mean total costs of the ELA and STA groups represents the incremental cost: $\$ 642.2$ (ELA over STA). The mean gained QALYs and ICERs of ELA over STA was 0.02 and $\$ 32110.00 /$ QALY. With $3 \%$ and $5 \%$ annual discounts, the ICERs of ELA were $\$ 32108.40 /$ QALY and $\$ 32126.05 /$ QALY in the STA group, respectively (Table 5).

\section{Discussion}

Up to now, the optimal treatment of displaced intra-articular calcaneus fractures remains controversial even though the equipment and technologies have been developing rapidly. The ELA can provide good visualization of the fracture site but several studies have reported that the postoperative wound complications rate including wound edge necrosis, dehiscence, haematoma or infection is relatively high. The STA can minimize soft tissue damage and reduce the risk of postoperative complication while allowing comparative fracture reduction. Previous studies comparing the therapeutic efficacy and clinical outcomes of ORIF via ELA or STA have showed no significant differences. ${ }^{37-40}$ From our results, the clinical outcomes including the Bohler's and Gissane angle, VAS scores, AOFAS scores were in accord with previous studies. Nevertheless, to the best of our knowledge, no studies comparing the direct costs and effectiveness of two techniques in China were conducted. Therefore, we have conducted this costutility analysis and provide another perspective for surgeons to make an optimally clinical decision in economic perspective.

In recent decades, surgery for treating calcaneus fractures has showed that it can bring great costeffectiveness compared with nonoperative treatments. For both surgeons and patients, they have high requirements of satisfaction on clinical outcomes and cost-effectiveness due to the advancement of medicine and therefore, costs are a crucial factor during making clinical decision. Cost-analysis is attached great significance of making clinical decision and is frequently used to evaluate which intervention can offer figure through comparing the cost and health impact of interventions. ${ }^{41}$ Thus, surgeons are supposed to make full assessments of costs and take benefits into consideration when making clinical strategy to manage specific patients. ${ }^{42}$

An article published in 2017 has used pooled data of western country to compare the cost and benefits in patients with calcaneus fractures classified as Sanders type II/III, which were managed with surgeries via extensile lateral approach or sinus tarsi approach or non-operative treatments. ${ }^{32}$ It demonstrated that ORIF via STA is the least expensive option for treating Sanders type II/III concerning total costs, probability of working the same job and duration out of work after ORIF. However, previous study did not conduct systematic retrospective review and report itemized details of the costs, which may hamper the ability to draw firm conclusions about cost-effectiveness by limited data. Moreover, long-term results after 
newer or refine ORIF techniques are unknown. In our study, we collected and checked the medical bills from single information center of all patients in order to conduct a Cost-Analysis.

In this retrospective view, only direct health costs were collected, and for the indirect health costs including miss time from work or decreased productivity, rehabilitation, further consultation and transportation were not calculated. CUA can be performed even without indirect costs. ${ }^{43-45}$ According to the results, we found no statistical differences in total costs, laboratory and radiographic evaluation expense, surgical expenses, antibiotic drugs expense, anesthesia expense and the length of hospital stay. Our study found significant differences in the analgesia expenses and internal fixation materials costs. The costs of patients in ELA group were higher than the cost of patients in STA group $\$ 145.8 \pm 85.6$ versus $\$ 102.9 \pm 62.7, P=.080$ ). Patients underwent surgical treatment via ELA have caused much more severe injury to soft tissue and blood vessels comparing with STA. Owing to the larger wound caused by ELA, patients' complaints of pain in the ELA group were more obvious. According to the pain measurement and VAS scores, there did exist significant difference in scores at 3 days and 5 days after surgery. Therefore, clinical strategies were made including using more effective analgesic drugs or extending the time of applying analgesic drugs, which resulted in the statistical difference in the expense of analgesia between these two groups. However, after postoperative treatments and caring, there showed no significant difference in VAS scores at 7 days after surgery.

Concerning the surgeon opinion for both technique, surgeon A could master ELA and STA techniques proficiently while surgeon B prefers performing ELA technique and surgeon C prefers performing STA technique. Additionally, patients' specific condition and subjective wishes are supposed to be taken into consideration and therefore, there were no significant difference among surgeon rating for both surgical techniques to reconstruct the height, width, Bohler's and Gissane angle of the calcaneus, therefore reaching the anatomical reduction. In this study, the brand of materials used in ELA group consists of Smith \& nephew (29/62), Acumend (10/62) and Double medical technology Inc-China (23/62) while in STA group consists of Acumed (31/47) and Carefix-China (16/47). Normally, the option of choosing which type of plates mainly depend on patients' condition and surgeon's preferences. However, healthcare system varies in different countries and it can be assumed that there is some bias in choosing internal fixation materials based on patient income and insurance type. When conditions permit, surgeons provide patients with suggestions on therapeutic scheme patients and patients can choose imported or domestic internal fixation materials according to their financial status and wishes. Currently, domestic internal fixation materials have a price advantage and can reach similarly anatomical reduction with low complication rate compared with imported ones. In our study, the patients in the ELA group underwent ORIF with plates and five to eight 3.5-mm screws while the patients in the STA group underwent ORIF with three to four $6.5-\mathrm{mm}$ cannulated screws solely. In the past 20 years, the technique of STA has been introduced in China and there were no comparable implants to match it and therefore this technique has not been popularized. Currently, as new generation of implants emerge, more and more surgeons tend to perform minimal invasive incisions and the cost of these implants are comparable to plates system in extensile lateral technique. Whether plate system or screws are applied via two different approaches, 
there were no definite indications for it. Based on our experience, normally procedures via ELA do not apply with screws solely and procedures via STA do not apply with large plates. We tend to apply screws for patients with intact calcaneal wall and simple posterior calcaneal articular surface collapse. For those patients with factures of calcaneal anterior process or impingement of fracture fragments, we tend to apply plate systems to reach anatomic reduction. The selection of implants is based on the specific condition of each patients and we control other parameters including patients' information and fracture type and that is the way we justified the cost of the patients.

As for the life of various type of implants, normally the implants will be removed if situations including pain, infections and etc. happen. In our countries, a majority of patients choose to have their implants removed as long as the fracture site reaches clinical healing standard or 24 months after the surgery. However, it may not be possible to track and analyze the cost of removal internal fixations for it may not be performed in the same hospital.

In our study, no severe complications occurred and all the patients at final follow up observations showed an excellent therapeutic effect after surgery according to direct measurement of clinical outcomes. As the results showed that there were no significant differences between the ELA and STA group among different surgeons on the clinical efficacy indicators. Changes in pre- and postoperative VAS and AOFAS scores are both the most direct measurement and rapid evidence of surgical outcomes. Altogether, the application of surgical incision on ELA or STA among surgeon A, B and C depends on their own experience and preference. According to the clinical indicators, both incisions have comparative curative effects and equivalent precision ratio on patients with type II/III calcaneus fracture because there were no significant differences. They can alleviate patients' pain and enable them to take their own responsibilities in the society.

The outcome of CUA was presented with gained QALYs, which were enumerated through multiplying the length of hospital stay by HRQoL weight (i.e, utility score) scaled from -0.11 to $1.00 .{ }^{46}$ Direct elicitation methods, generic preference-based measures, and condition-specific measures were used to evaluate HRQoL. The EQ-5D-3L, regarded as the primary valuation study derived utility scores, are prevalently applied preference-based measures. ${ }^{47-49}$ In this study, CER and ICERs were the outcomes of economic benefits, which could be comprehended that ICERs indicate how much extra health benefits via ELA can bring compared with via STA, and how much additional expense it will cost.

Consequently, we found no statistically significant differences in overall cost per patient and HRQoL between two groups but generally surgery via ELA incurred much costs than via STA. Similarly, the incremental cost-utility ratio also showed there were no cost-utility benefits comparing two groups.

\section{Limitation}

This study has its own limitations. Firstly, the chief limitation of study is that it is a retrospective costutility analysis and randomized clinical trials (RCTs) are insufficient. Therefore, there are inherent defect 
existing in the study. Secondly, all the data are collected from a sing center, which leads to the fact that the sample size is not adequate and it may result in single center analysis bias. Thirdly, indirect costs including rehabilitation, home care and further consultation have not been collected as patients may go back to local hospital and produce fees that could not be tracked. Fourthly, only patients classified as Sanders type II/III were focused solely on in the study and future RCTs combined with CUA could emphasis on other types of calcaneus fractures such as Sanders I/IV, malunion and nonunion. Lastly, there did exist some level of recall bias in patients accomplished questionnaires and limitation of all CUA in lacking of uniform methodology to track the preoperative outcome.

\section{Conclusion}

Both ELA and STA techniques are effective surgical procedures for the patients with Calcaneus Fracture. Moreover, STA seems to be more reasonable for its merits including less postoperative pain, fewer expenses of analgesia and internal fixation materials.

\section{Declarations}

\section{Acknowledgements}

Zihua Li, Xinbo Wu and Haichao Zhou are co-first authors.

\section{Authors' contributions}

$\mathrm{LZH}, \mathrm{WXB}$, and ZHC conceived and designed the study. XSC, XFJ, and $\mathrm{HH}$ measured and recorded the data. $\mathrm{LZH}$ and ZHC wrote the paper. WXB and YYF reviewed and edited the manuscript. All authors read and approved the manuscript.

\section{Funding}

1. National Natural Science Foundation of China (no. 31800782)

\section{Availability of data and materials}

The datasets used and/or analyzed during the current study are available from the corresponding author upon reasonable request.

\section{Ethics approval and consent to participate}

This retrospective study was approved and consented to participate by the Ethics Committee.

\section{Consent for publication}

Not applicable. 


\section{Competing interests}

The authors declare that they have no competing interests.

\section{Author details}

Department of Orthopedics, Shanghai Tongji Hospital, Tongji University School of Medicine, Shanghai 200065, China

\section{Abbreviations}

ELA: Extensile Lateral Approach; STA: Sinus Tarsi Approach;

DIACFs: Displaced intra-articular calcaneus fractures; CUA: Cost-utility analysis;

ORIF: Open reduction and internal fixation;

ICERs: incremental cost-effectiveness ratio; HRQoL: Health-related quality of life;

VAS: Visual analogue scale; EQ-5D: EuroQol five-dimensional;

AOFAS: American Orthopaedic Foot and Ankle Society; QALY: quality-adjusted life-year.

\section{References}

1. CM Court-Brown, Caesar B. Epidemiology of adult fractures: A review. Injury 2006;37(8):691-7.

2. H Zwipp, Rammelt S, Barthel S. [Fracture of the calcaneus]. Der Unfallchirurg 2005;108(9):737-47; quiz 748.

3. T Schepers, van Lieshout EM, van Ginhoven TM, Heetveld MJ, Patka P. Current concepts in the treatment of intra-articular calcaneal fractures: results of a nationwide survey. International orthopaedics 2008;32(5):711-5.

4. D Griffin, Parsons N, Shaw E, et al. Operative versus non-operative treatment for closed, displaced, intra-articular fractures of the calcaneus: randomised controlled trial. BMJ (Clinical research ed.) 2014;349(undefined):g4483.

5. Ocguder A., H. Gok, C. Heycan, O. Tecimel, E. Tonuk, M. Bozkurt. Effects of custom-made insole on gait pattern of patients with unilateral displaced intra-articular calcaneal fracture: evaluation with computerized gait analysis. Acta Orthop Traumatol Turc 2012;46(1):1-7. doi: 10.3944/aott.2012.2401.

6. R Sanders, Fortin P, DiPasquale T, Walling A. Operative treatment in 120 displaced intraarticular calcaneal fractures. Results using a prognostic computed tomography scan classification. Clinical orthopaedics and related research 1993;undefined(290):87-95. 
7. Sanders R. Displaced intra-articular fractures of the calcaneus. J Bone Joint Surg Am 2000;82(2):225-50. doi: 10.2106/00004623-200002000-00009. doi: 10.2106/00004623-20000200000009 .

8. MP Clare, Lee WE, Sanders RW. Intermediate to long-term results of a treatment protocol for calcaneal fracture malunions. The Journal of bone and joint surgery. American volume 2005;87(5):963-73.

9. Crosby L. A., T. Fitzgibbons. Intraarticular calcaneal fractures. Results of closed treatment. Clin Orthop Relat Res 1993;\&NA;(290):47-54.

10. Cottom J. M., J. S. Baker. Restoring the Anatomy of Calcaneal Fractures: A Simple Technique With Radiographic Review. Foot Ankle Spec 2017;10(3):235-239. doi: 10.1177/1938640016679700.

11. D Makki, Alnajjar HM, Walkay S, Ramkumar U, Watson AJ, Allen PW. Osteosynthesis of displaced intra-articular fractures of the calcaneum: a long-term review of 47 cases. The Journal of bone and joint surgery. British volume 2010;92(5):693-700.

12. T Ibrahim, Rowsell M, Rennie W, Brown AR, Taylor GJ, Gregg PJ. Displaced intra-articular calcaneal fractures: 15-year follow-up of a randomised controlled trial of conservative versus operative treatment. Injury 2007;38(7):848-55.

13. TD Tennent, Calder PR, Salisbury RD, Allen PW, Eastwood DM. The operative management of displaced intra-articular fractures of the calcaneum: a two-centre study using a defined protocol. Injury 2001;32(6):491-6.

14. Benirschke S. K., P. A. Kramer. Wound healing complications in closed and open calcaneal fractures. J Orthop Trauma 2004;18(1):1-6. doi: 10.1097/00005131-200401000-00001.

15. Al-Mudhaffar M., C. V. Prasad, A. Mofidi. Wound complications following operative fixation of calcaneal fractures. Injury 2000;31(6):461-4. doi: 10.1016/s0020-1383(00)00026-7.

16. Folk Jw. Early wound complications of operative treatment of calcaneus fractures : analysis of 190 fractures. Journal of Orthopaedic Trauma 1994;13.

17. Kline A. J., R. B. Anderson, W. H. Davis, C. P. Jones, B. E. Cohen. Minimally invasive technique versus an extensile lateral approach for intra-articular calcaneal fractures. Foot Ankle Int 2013;34(6):773-80. doi: 10.1177/1071100713477607.

18. Herscovicijr Dolfi. Operative Treatment of Calcaneal Fractures in Elderly Patients. 2005;87(6):12601264.

19. MF Mostafa, El-Adl G, Hassanin EY, Abdellatif MS. Surgical treatment of displaced intra-articular calcaneal fracture using a single small lateral approach. Strategies in trauma and limb reconstruction 2010;5(2):87-95.

20. JE Femino, Vaseenon T, Levin DA, Yian EH. Modification of the sinus tarsi approach for open reduction and plate fixation of intra-articular calcaneus fractures: the limits of proximal extension based upon the vascular anatomy of the lateral calcaneal artery. The lowa orthopaedic journal 2010;30(undefined):161-7. 
21. Gupta A., N. Ghalambor, A. Nihal, E. Trepman. The modified Palmer lateral approach for calcaneal fractures: wound healing and postoperative computed tomographic evaluation of fracture reduction. Foot Ankle Int 2003;24(10):744-53. doi: 10.1177/107110070302401003.

22. Tao Zhang, Yanling Su, Chen Wei, Zhang Qi, Zhanpo Wu, Yingze Zhang. Displaced Intra-Articular Calcaneal Fractures Treated in a Minimally Invasive Fashion. 2014;96(4):302-309.

23. Xia S., Y. Lu, H. Wang, Z. Wu, Z. Wang. Open reduction and internal fixation with conventional plate via $L$-shaped lateral approach versus internal fixation with percutaneous plate via a sinus tarsi approach for calcaneal fractures - a randomized controlled trial. Int J Surg 2014;12(5):475-80. doi: 10.1016/j.ijsu.2014.03.001.

24. AJ Kline, Anderson RB, Davis WH, Jones CP, Cohen BE. Minimally invasive technique versus an extensile lateral approach for intra-articular calcaneal fractures. Foot \& ankle international 2013;34(6):773-80.

25. JH Yeo, Cho HJ, Lee KB. Comparison of two surgical approaches for displaced intra-articular calcaneal fractures: sinus tarsi versus extensile lateral approach. BMC musculoskeletal disorders 2015;16(undefined):63.

26. T Schepers. The sinus tarsi approach in displaced intra-articular calcaneal fractures: a systematic review. International orthopaedics 2011;35(5):697-703.

27. Bozkurt M., B. B. Kentel, G. Yavuzer, A. Ocguder, C. Heycan, E. Tonuk. Functional evaluation of intraarticular severely comminuted fractures of the calcaneus with gait analysis. J Foot Ankle Surg 2004;43(6):374-9. doi: 10.1053/j.jfas.2004.09.006.

28. Hirschmüller Anja, Lukas Konstantinidis, Heiner Baur, et al. Do changes in dynamic plantar pressure distribution, strength capacity and postural control after intra-articular calcaneal fracture correlate with clinical and radiological outcome? 2011;42(10):1135-1143.

29. L Besch, Radke B, Mueller M, et al. Dynamic and functional gait analysis of severely displaced intraarticular calcaneus fractures treated with a hinged external fixator or internal stabilization. The Journal of foot and ankle surgery : official publication of the American College of Foot and Ankle Surgeons 2008;47(1):19-25.

30. Contreras Marcos Emilio Kuschnaroff, Adriane Mara De Souza Muniz, Juliana Barcellos De Souza, et al. Biomechanical evaluation of intra articular calcaneal fracture and clinical radiographic correlation. 2004;12(2):105-112.

31. Albin S. R., M. W. Cornwall, T. G. McPoil, D. H. Van Boerum, J. M. Morgan. Plantar Pressure and Gait Symmetry in Individuals with Fractures versus Tendon Injuries to the Hindfoot. J Am Podiatr Med Assoc 2015;105(6):469-77. doi: 10.7547/14-073.1.

32. RC Clement, Lang PJ, Pettett BJ, Overman RA, Ostrum RF, Tennant JN. Sanders II/III Calcaneus Fractures in Laborers: A Cost-Effectiveness Analysis and Call for Effectiveness Research. Journal of orthopaedic trauma 2017;31(6):299-304.

33. GD Sanders, Neumann PJ, Basu A, et al. Recommendations for Conduct, Methodological Practices, and Reporting of Cost-effectiveness Analyses: Second Panel on Cost-Effectiveness in Health and 
Medicine. JAMA 2016;316(10):1093-103.

34. LB Russell, Gold MR, Siegel JE, Daniels N, Weinstein MC. The role of cost-effectiveness analysis in health and medicine. Panel on Cost-Effectiveness in Health and Medicine. JAMA 1996;276(14):11727.

35. A Manca, Hawkins N, Sculpher MJ. Estimating mean QALYs in trial-based cost-effectiveness analysis: the importance of controlling for baseline utility. Health economics 2005;14(5):487-96.

36. G Richardson, Manca A. Calculation of quality adjusted life years in the published literature: a review of methodology and transparency. Health economics 2004;13(12):1203-10.

37. N Jiang, Lin QR, Diao XC, Wu L, Yu B. Surgical versus nonsurgical treatment of displaced intraarticular calcaneal fracture: a meta-analysis of current evidence base. International orthopaedics 2012;36(8):1615-22.

38. F Zhang, Tian H, Li S, et al. Meta-analysis of two surgical approaches for calcaneal fractures: sinus tarsi versus extensile lateral approach. ANZ journal of surgery 2017;87(3):126-131.

39. N Wei, Yuwen P, Liu W, et al. Operative versus nonoperative treatment of displaced intra-articular calcaneal fractures: A meta-analysis of current evidence base. Medicine 2017;96(49):e9027.

40. X Luo, Li Q, He S, He S. Operative Versus Nonoperative Treatment for Displaced Intra-Articular Calcaneal Fractures: A Meta-Analysis of Randomized Controlled Trials. The Journal of foot and ankle surgery : official publication of the American College of Foot and Ankle Surgeons 2016;55(4):821-8.

41. SD Grosse, Teutsch SM, Haddix AC. Lessons from cost-effectiveness research for United States public health policy. Annual review of public health 2007;28(undefined):365-91.

42. SM Teutsch, Murray JF. Dissecting cost-effectiveness analysis for preventive interventions: a guide for decision makers. The American journal of managed care 1999;5(3):301-5.

43. EY Bae. [Guidelines for economic evaluation of pharmaceuticals in Korea]. Journal of preventive medicine and public health = Yebang Uihakhoe chi 2008;41(2):80-3.

44. Glennie J. L., G. W. Torrance, J. F. Baladi, et al. The revised Canadian Guidelines for the Economic Evaluation of Pharmaceuticals. Pharmacoeconomics 1999;15(5):459-68. doi: 10.2165/00019053$199915050-00004$.

45. Torrance G. W., D. Blaker, A. Detsky, et al. Canadian guidelines for economic evaluation of pharmaceuticals. Canadian Collaborative Workshop for Pharmacoeconomics. Pharmacoeconomics 1996;9(6):535-59. doi: 10.2165/00019053-199609060-00008.

46. SJ Whitehead, Ali S. Health outcomes in economic evaluation: the QALY and utilities. British medical bulletin 2010;96(undefined):5-21.

47. Coretti S., M. Ruggeri, P. McNamee. The minimum clinically important difference for EQ-5D index: a critical review. Expert Review of Pharmacoeconomics \& Outcomes Research 2014;14(2):221-233. doi: 10.1586/14737167.2014.894462. 
48. F Xie, Gaebel K, Perampaladas K, Doble B, Pullenayegum E. Comparing EQ-5D valuation studies: a systematic review and methodological reporting checklist. Medical decision making : an international journal of the Society for Medical Decision Making 2014;34(1):8-20.

49. N Payakachat, Ali MM, Tilford JM. Can The EQ-5D Detect Meaningful Change? A Systematic Review. PharmacoEconomics 2015;33(11):1137-54.

\section{Tables}

TABLE 1. Patient Information

\begin{tabular}{|c|c|c|c|}
\hline Parameters & ELA $(N=62)$ & STA $(N=47)$ & $p$ value \\
\hline$\overline{\text { Age(year) }}$ & $45.40 \pm 12.91$ & $49.92 \pm 14.98$ & .095 \\
\hline Gender & & & .522 \\
\hline Male & 51 & 38 & \\
\hline Female & 11 & 9 & \\
\hline Fracture type & & & .516 \\
\hline Sanders II & 36 & 28 & \\
\hline Sanders III & 26 & 19 & \\
\hline Anesthesia & & & .860 \\
\hline Spinal anesthesia & 52 & 40 & \\
\hline General anesthesia & 10 & 7 & \\
\hline the Length of hospital stay & $10.9 \pm 3.7$ & $9.4 \pm 3.6$ & .060 \\
\hline Follow-up time(month) & $30.5 \pm 9.7$ & $28.4 \pm 13.5$ & .150 \\
\hline
\end{tabular}

Data are means \pm SD and numbers of subjects. Significant difference $(\mathrm{P}<.05)$.

ELA: extensile lateral approach; STA: sinus tarsi approach. 
TABLE 2. Clinical Outcome

\begin{tabular}{llll}
\hline Parameters & ELA $(N=62)$ & STA $(N=47)$ & $p$ value
\end{tabular}

VAS scores

$\begin{array}{llll}\text { Preoperative } & 6.9 \pm 1.1 & 6.6 \pm 1.0 & .151 \\ \text { Postoperative 3 days } & 4.6 \pm 0.9 & 3.0 \pm 0.8 & \square .001 \\ \text { Postoperative 5 days } & 2.3 \pm 0.7 & 1.8 \pm 0.5 & .002 \\ \text { Postoperative 7 days } & 1.6 \pm 0.5 & 1.5 \pm 0.5 & .693\end{array}$

AOFAS

Preoperative

$50.0 \pm 8.0$

$51.2 \pm 7.9$

.413

Postoperative 3 months

$80.3 \pm 6.3$

$81.4 \pm 5.6$

.325

Final follow-up

$83.7 \pm 4.6$

$85.0 \pm 4.0$

.113

Bohler's angle(degrees)

Pre-operation

$13.6 \pm 7.3$

$16.0 \pm 7.4$

.086

Postoperative 3 months

$30.2 \pm 7.6$

$28.2 \pm 7.1$

.053

Final follow-up

$29.9 \pm 6.6$

$28.4 \pm 6.3$

.065

Gissane angle(degrees)

Pre-operation

$102.8 \pm 14.2$

$102.0 \pm 12.5$

.920

Postoperative 3 months

$130.0 \pm 9.2$

$127.7 \pm 8.2$

.255

Final follow-up

$126.0 \pm 7.5$

$128.2 \pm 7.8$

.139

Preoperative EQ-5D score

$0.63(0.57-0.68)$

$0.59(0.52-0.68)$

.246

Postoperative EQ-5D

0.92 (0.89-0.95)

$0.93(0.88-0.95)$

.755

Gained QALY

$0.77(0.73-0.80)$

$0.75(0.69-0.79)$

.250

EQ-5D: EuroQol five-dimensional; VAS: Visual Analogue Scale; AOFAS: American Orthopaedic Foot and Ankle Society; QALY: quality-adjusted life-year. 
TABLE 3. Health Care Cost

\begin{tabular}{llll}
\hline Parameters & ELA $(N=62)$ & STA $(N=47)$ & $p$ value \\
\hline Laboratory expense & $\$ 143.1 \pm 42.9$ & $\$ 160.8 \pm 109.5$ & .605 \\
Radiography & $\$ 78.1 \pm 21.8$ & $\$ 80.8 \pm 15.1$ & .217 \\
CT & $\$ 46.3 \pm 21.8$ & $\$ 44.5 \pm 21.7$ & .506 \\
\hline Surgery & $\$ 396.3 \pm 130.5$ & $\$ 442.9 \pm 202.7$ & .376 \\
Anesthesia & $\$ 146.3 \pm 67.5$ & $\$ 142.0 \pm 70.1$ & .778 \\
Analgesia & $\$ 145.8 \pm 85.6$ & $\$ 102.9 \pm 62.7$ & .008 \\
Internal Fixation Materials & $\$ 3289.0 \pm 543.9$ & $\$ 2630.6 \pm 763.7$ & 0.001 \\
Antiboitic drugs & $\$ 85.0 \pm 44.6$ & $\$ 76.5 \pm 54.9$ & .104 \\
Total cost & $\$ 6481.0 \pm 1504.4$ & $\$ 5838.8 \pm 1276.2$ & .200 \\
\hline
\end{tabular}

Significant difference (Р.05). CT: Computed tomography; Values represent the mean cost per patient in January 2020 US Dollars. 
TABLE 4. Cost-effectiveness Ratios (CERs)

\begin{tabular}{lccc}
\hline Parameters & ELA $(N=62)$ & STA $(N=47)$ & $p$ value \\
\hline Cost & $\$ 6481.0 \pm 1504.4 \$ 5838.8 \pm 1276.2$ & .200 \\
Utility (QALY) & $0.77(0.73-0.80)$ & $0.75(0.69-0.79)$ & .755 \\
CERs & $\$ 8766.8 \pm 2835.2 \$ 7914.9 \pm 1822.0$ & .245
\end{tabular}

QALY:quality-adjusted life-year.

TABLE 5. Incremental Cost-effectiveness Ratio (ICER)

\begin{tabular}{lccc}
\hline Parameters & Normal & \multicolumn{3}{c}{$3 \%$ Discount5\% Discount } \\
\hline$\Delta$ Cost & 642.20 & 623.51 & 605.36 \\
$\Delta$ QALY & 0.02 & $1.942 \times 10^{-2}$ & $1.94 \times 10^{-2}$ \\
\hline ICER $(\Delta$ cost/ $\Delta$ QALY) 32110.00 & 32106.59 & 31794.26 \\
\hline
\end{tabular}

ICER: incremental cost-effectiveness ratio. 


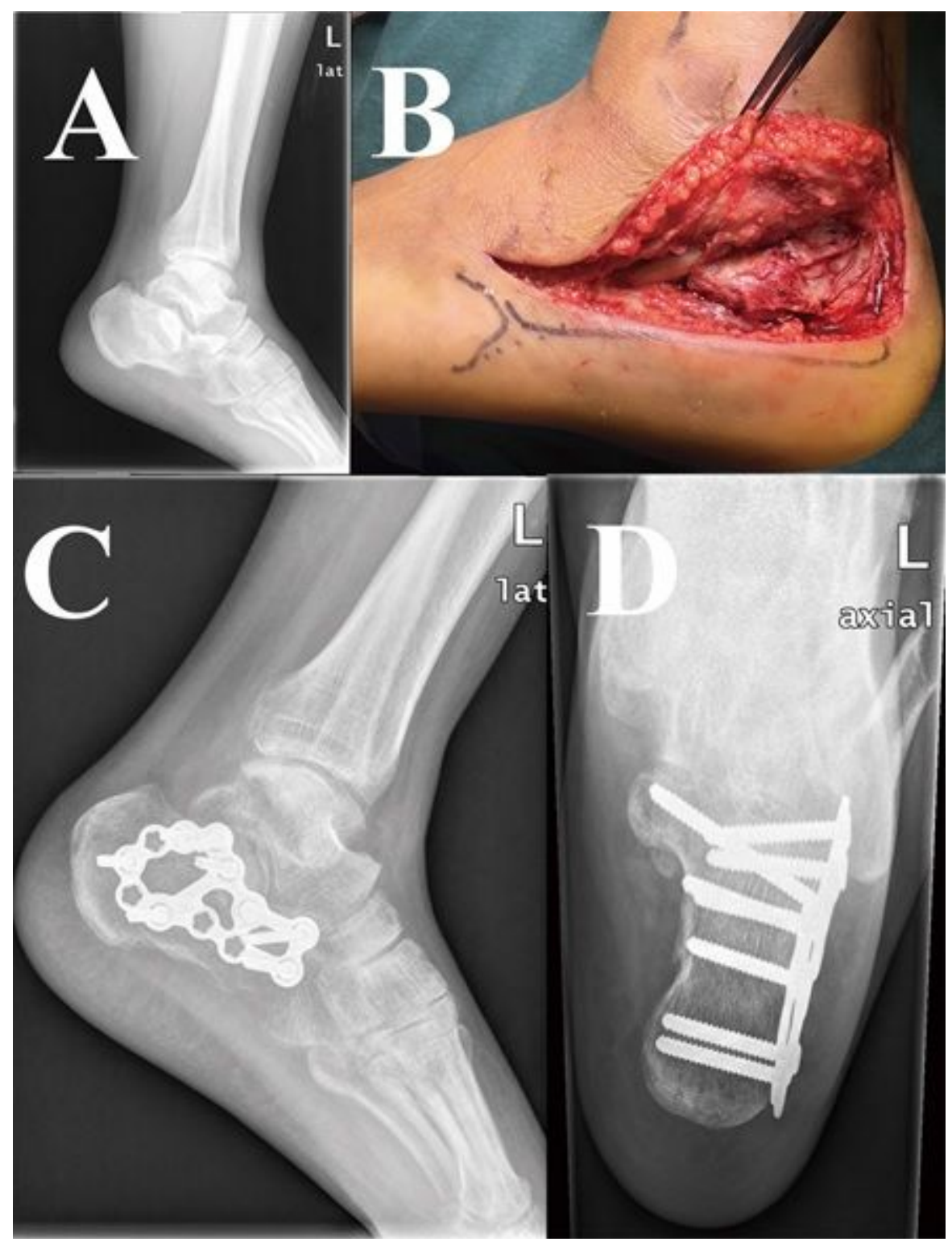

Figure 1

A) The preoperative lateral image demonstrates collapse of the Bohler's angle and posterior facet. (B) Intraoperative gross image of surgery via the extensile lateral approach (ELA). (C) The postoperative lateral image shows restoration of the Bohler's angle and posterior facet by plate and screws. (D) The axial image shows restoration of the width and alignment of calcaneus. 


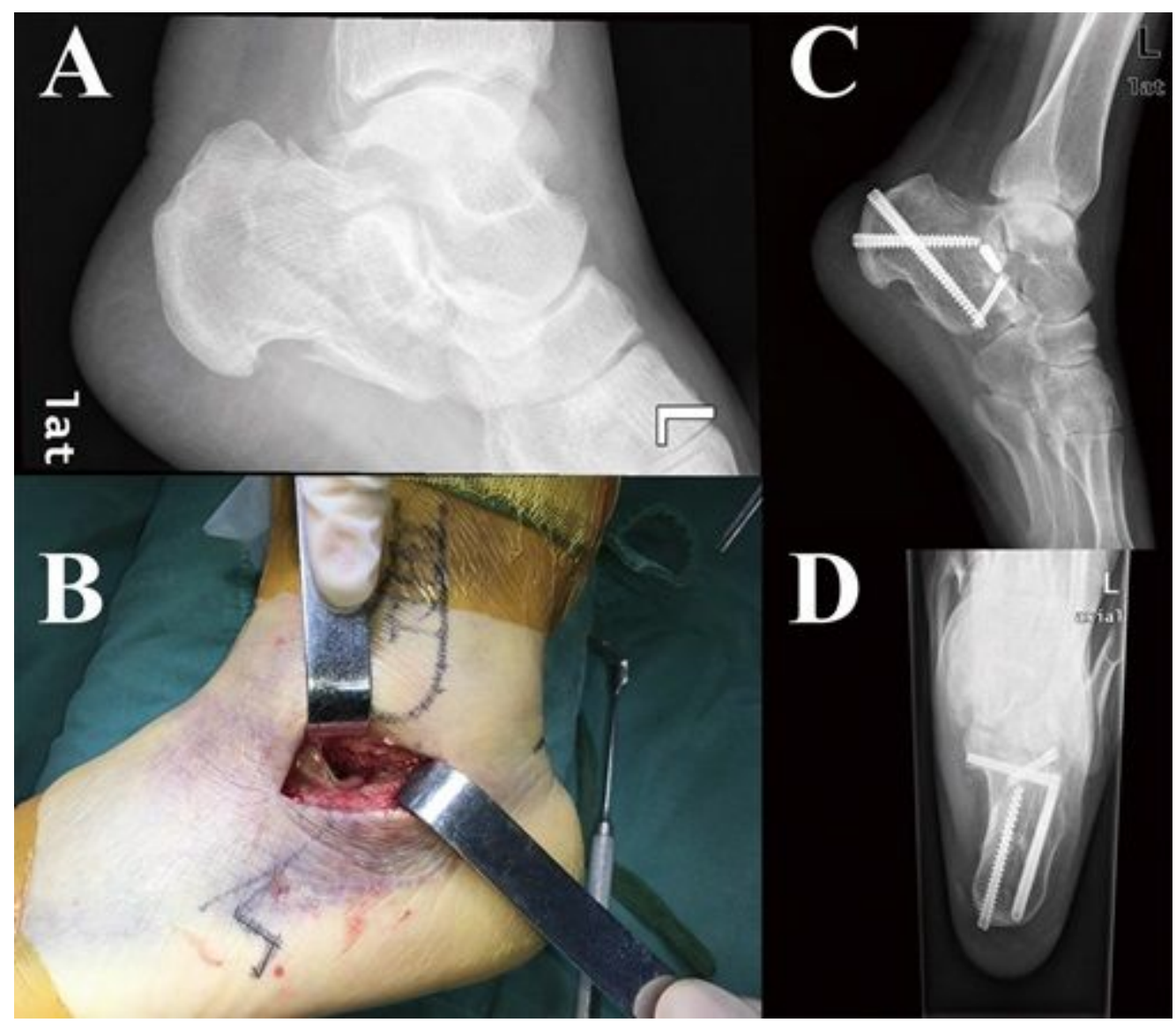

Figure 2

(A) The preoperative lateral image shows fracture line involving the subtalar joint. (B) Intraoperative gross image of surgery via the sinus tarsi approach (STA). (C) The postoperative lateral image shows restoration of Bohler's angle and fracture line involving subtalar joint was reduced by screws. (D) The axial image shows the normal alignment of calcaneus.

\section{Supplementary Files}

This is a list of supplementary files associated with this preprint. Click to download.

- formula.docx 